\title{
Robust Control of STATCOM Based on Sliding Mode Technique
}

\author{
M. Abbasian \\ Department of Engineering \\ Islamic Azad University- Khorasgan Branch \\ Isfahan (Iran) \\ Phone/Fax number:+98 311 5354001, e-mail: a_abbasian@ec.iut.ac.ir
}

\begin{abstract}
This paper presents a robust controller for GTO based static compensators (STATCOM), which guarantees fast and stable voltage regulation under all operating conditions. The design of control system is based upon the Sliding Mode technique. The system is simulated in order to validate the controller performances. The stability of proposed composite controller is proved by Lyaponuv theory. The effectiveness and validity of the controller system is supported by computer simulation. Simulation results obtained, confirm that the controller is robust and stable against all of the parameters variations and uncertainties.
\end{abstract}

\section{Key words}

STATCOM, FACTS, Robust Control, Sliding Mode.

\section{Introduction}

Flexible AC transmission Systems (FACTS) devices are a very innovative and interesting application of power electronics to the control of power networks. Current advances in power electronics devices now allow the use of new generation of FACTS devices based on the selfcommutated voltage-sourced converter (VSC) using Gate-Turn-off thyristor technology. It includes the Static Compensator (STATCOM), Series Power Flow Controller (SPFC), VSC-based Static Phase Shifter (SPS) and Unified Power Flow Controller (UPFC) [1-3].

STATCOM has been employed to an increasing extent in modem power systems due to its capability to work as var generation and absorption systems. Besides the main task of voltage control, STATCOM may also be applied to improve the transmission capability and stability.

In order to gain as much as possible the benefits from these devices for local voltage support and stability enhancement of the global systems, various control methods have been studied for these power components. Conventional controllers for instance are usually designed to have fast response and adequate stability margin at the weakest network condition. For example, the PID controller is simple and is by far, the most widely used control scheme in the process industry. However, the controller gains are determined under a particular operating condition and may become completely unsatisfactory for another [4].

The nonlinear nature of STATCOM and The dynamics of an electric power system that are subject to changes due to lightning, storms, equipment failures, and so on, make it difficult to design a controller that guarantees fast and stable voltage regulation under all operating conditions. The challenge is to find a design method which is global and is independent of the operating point. Nonlinear control is one answer to this search.

Reference [5] proposes a control scheme based on inputoutput linearization technique to improve transient performances of the compensators. Reference [6] proposes a control scheme based on input-output linearization technique to improve transient performance of the compensator. In [7] another linearization technique is used, but the proposed condoler is not robust against the STATCOM and power system uncertainties.

It is thus imperative that we design a controller that can provide satisfactory performance under a wide range of operating conditions.

It is well known that the sliding mode control technique provides superior robustness performance in terms of system model variations compared to the linear controllers.

The objective of this work is to examine a robust controller for STATCOM based on Sliding Mode Method which is robust against all parameter uncertainties. In this paper a new modeling is introduced and used to control STATCOM using Sliding-Mode method.

\section{System Modeling and Control}

Studied system (Figure 1) consists of a utility power system and a self commutated inverter that is capable of compensating reactive power. This inverter essentially comprises six self commutated thyristors, such as GTO with antiparallel diode. The control signal is the pulse width modulation (PWM) switching sequence. 


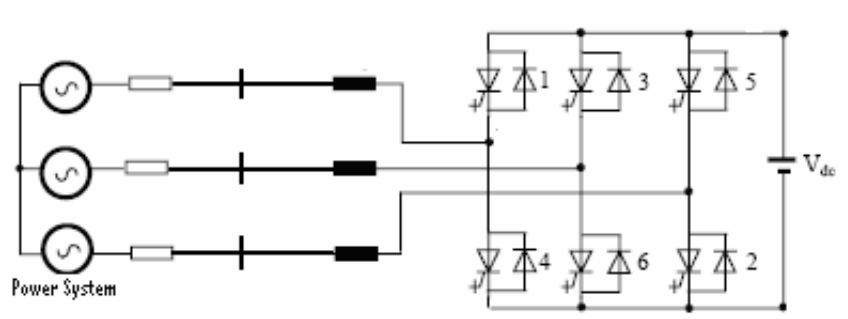

Fig. 1. Proposed Power Circuit

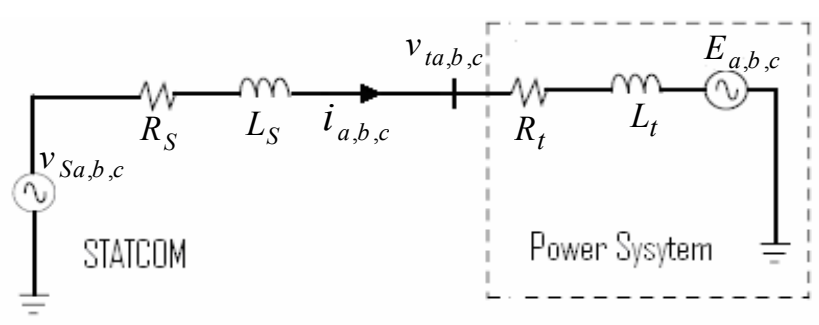

Fig. 2. Equivalent circuit

This paper presents a new modeling and a robust controller for STATCOM. The modeling is based on the $a-b-c$ to $d-q$ transformation of the ac system variables. The voltage supplied by the STATCOM is controlled in the synchronous orthogonal $d-q$ frame using a robust control strategy.

Figure 2 shows the equivalent circuit of a PWM Converter connected as a STATCOM. $R_{t}$ and $L_{t}$ are the equivalent Thevenin resistance and inductance of the power system as seen by the STATCOM and $E_{a, b, c}$ is the equivalent Thevenin voltage. In addition, $v_{S a, b, c}, R_{S}$ and $L_{S}$, represent STATCOM model including the coupling transformer's (Figure 3) resistance and inductance. $v_{S a, b, c}$ is the output voltage of STATCOM and the input voltage of the coupling transformer. $v_{t a, b, c}$ is the terminal voltage (output voltage of the coupling transformer) that should be controlled. It is assumed that the terminal is feeding a variable load.

After applying Kirchhoff's Voltage Law to this circuit and transforming the equations to synchronous orthogonal d-q frame, a direct relationship between input and output is achieved, that would be described below.

At first, the 3-phase current equations can be written as follows:

$$
\begin{aligned}
{\left[\begin{array}{l}
v_{S a} \\
v_{S b} \\
v_{S c}
\end{array}\right]=} & {\left[\begin{array}{l}
v_{t a} \\
v_{t b} \\
v_{t c}
\end{array}\right]+\left[\begin{array}{ccc}
R_{S} & 0 & 0 \\
0 & R_{S} & 0 \\
0 & 0 & R_{S}
\end{array}\right]\left[\begin{array}{l}
i_{a} \\
i_{b} \\
i_{c}
\end{array}\right] } \\
& +L_{S} \frac{d}{d t}\left[\begin{array}{l}
i_{a} \\
i_{b} \\
i_{c}
\end{array}\right]
\end{aligned}
$$

$$
\begin{aligned}
{\left[\begin{array}{l}
v_{t a} \\
v_{t b} \\
v_{t c}
\end{array}\right]=} & {\left[\begin{array}{l}
E_{a} \\
E_{b} \\
E_{c}
\end{array}\right]+\left[\begin{array}{lll}
R_{t} & 0 & 0 \\
0 & R_{t} & 0 \\
0 & 0 & R_{t}
\end{array}\right]\left[\begin{array}{l}
i_{a} \\
i_{b} \\
i_{c}
\end{array}\right] } \\
& +L_{t} \frac{d}{d t}\left[\begin{array}{l}
i_{a} \\
i_{b} \\
i_{c}
\end{array}\right]
\end{aligned}
$$

where $\omega$ is the angular velocity of ac voltage and current vectors. Equations (1) and (2) can be transferred to synchronously rotating $\mathrm{d}-\mathrm{q}$ reference frame as follows:

$$
\begin{aligned}
& {\left[\begin{array}{l}
v_{S d} \\
v_{S q}
\end{array}\right]=\left[\begin{array}{l}
v_{t d} \\
v_{t q}
\end{array}\right]+\left[\begin{array}{cc}
R_{S} & -\omega L_{S} \\
\omega L_{S} & R_{S}
\end{array}\right]\left[\begin{array}{l}
i_{d} \\
i_{q}
\end{array}\right]+L_{S} \frac{d}{d t}\left[\begin{array}{l}
i_{d} \\
i_{q}
\end{array}\right]} \\
& {\left[\begin{array}{l}
v_{t d} \\
v_{t q}
\end{array}\right]=\left[\begin{array}{l}
E_{d} \\
E_{q}
\end{array}\right]+\left[\begin{array}{cc}
R_{t} & -\omega L_{t} \\
\omega L_{t} & R_{t}
\end{array}\right]\left[\begin{array}{l}
i_{d} \\
i_{q}
\end{array}\right]+L_{t} \frac{d}{d t}\left[\begin{array}{l}
i_{d} \\
i_{q}
\end{array}\right]}
\end{aligned}
$$

Equations (3) and (4) can be written as:

$$
\begin{aligned}
& V_{S}=V_{t}+M I+L_{S} \frac{d I}{d t} \\
& V_{t}=E+N I+L_{t} \frac{d I}{d t}
\end{aligned}
$$

where:

$$
\begin{aligned}
& V_{S}=\left[\begin{array}{l}
v_{S d} \\
v_{S q}
\end{array}\right], V_{t}=\left[\begin{array}{l}
v_{t d} \\
v_{t q}
\end{array}\right], E=\left[\begin{array}{c}
E_{d} \\
E_{q}
\end{array}\right], I=\left[\begin{array}{l}
i_{d} \\
i_{q}
\end{array}\right] \\
& M=\left[\begin{array}{cc}
R_{S} & -\omega L_{S} \\
\omega L_{S} & R_{S}
\end{array}\right], N=\left[\begin{array}{cc}
R_{t} & -\omega L_{t} \\
\omega L_{t} & R_{t}
\end{array}\right]
\end{aligned}
$$

Equation (6) can be rewritten as:

$\frac{d I}{d t}=\frac{1}{L_{t}}\left(V_{t}-N I-E\right)$

Substituting equation (7) into equation (5) yiel ds:

$$
V_{S}=M I+\frac{L_{S}}{L_{t}}\left(V_{t}-N I-E\right)+V_{t}
$$

or:

$V_{S}=\left(M-\frac{L_{S}}{L_{t}} N\right) I+\left(\frac{L_{S}}{L_{t}}+1\right) V_{t}-\frac{L_{S}}{L_{t}} E$

using the following equation: 


$$
\begin{aligned}
M-\frac{L_{S}}{L_{t}} N & =\left[\begin{array}{cc}
R_{S} & -\omega L_{S} \\
\omega L_{S} & R_{S}
\end{array}\right]-\frac{L_{S}}{L_{t}}\left[\begin{array}{cc}
R_{t} & -\omega L_{t} \\
\omega L_{t} & R_{t}
\end{array}\right] \\
& =\left[\begin{array}{cc}
R_{S}-\frac{L_{S}}{L_{t}} R_{t} & 0 \\
0 & R_{S}-\frac{L_{S}}{L_{t}} R_{t}
\end{array}\right]
\end{aligned}
$$

Equation (9) can be rewritten as:

$$
V_{S}=\left(\frac{L_{S}}{L_{t}}+1\right) V_{t}+\left(R_{S}-\frac{L_{S}}{L_{t}} R_{t}\right) I-\frac{L_{S}}{L_{t}} E
$$

or:

$V_{S}=a V_{t}+b$

where:

$a=\frac{L_{S}}{L_{t}}+1, b=\left[\begin{array}{l}b_{1} \\ b_{2}\end{array}\right]=\left(R_{S}-\frac{L_{S}}{L_{t}} R_{t}\right) I-\frac{L_{S}}{L_{t}} E$

using equation (12), the final input-output system equation is obtained as:

$V_{t}=\alpha V_{S}+\beta$

where:

$\alpha=\frac{1}{a}, \beta=\left[\begin{array}{c}-\frac{b_{1}}{a} \\ -\frac{b_{2}}{a}\end{array}\right]$

In this system, $V_{S}$ is the control input, where $V_{t}$ is assumed to be the system output, hence we should design the control input so that the system output can track the desired trajectory, $V_{t}^{*}$, accurately. $V_{t}^{*}$ is the STATCOM terminal voltage command in synchronously rotating $\mathrm{d}-\mathrm{q}$ reference frame and can be defined as:

$V_{t}^{*}=\left[\begin{array}{c}v_{t d}^{*} \\ v_{t q}^{*}\end{array}\right]$

It is clear that there are uncertainties and variations in the power system parameters $\left(R_{t}, L_{t}, R_{S}, L_{S}\right.$ and $\left.E\right)$. So it is important to design a controller that is robust against all of the parameter uncertainties.

Using Sliding Mode technique, a robust controller is designed for this system which guarantees fast and stable voltage regulation under all operating conditions and parameters variations.

To design the robust controller, equation (14) is written as follows:
$V_{t}=\alpha V_{S}+\beta=\left(\bar{\alpha} V_{S}+\bar{\beta}\right)+\left(\Delta \alpha V_{S}+\Delta \beta\right)=Y+\psi$

where $Y=\left[\begin{array}{l}y_{d} \\ y_{q}\end{array}\right]=\bar{\alpha} V_{S}+\bar{\beta}$ is the new control input and

$\bar{\alpha}$ and $\bar{\beta}$ can be obtained from equation (15), using the nominal parameter values without considering the system parameter variations and uncertainties.

The lumped uncertainty vector is defined as:

$\psi=\left[\begin{array}{l}\psi_{d} \\ \psi_{q}\end{array}\right]=\Delta \alpha V_{S}+\Delta \beta$

in which $\Delta \alpha$ and $\Delta \beta$ denote the uncertainties introduced by mechanical and electrical parameters. The lumped uncertainties $\Delta \alpha$ and $\Delta \beta$ are assumed to be bounded, i.e.

The lumped uncertainties $\psi_{d}$ and $\psi_{q}$ are assumed to be bounded, i.e. $\left|\psi_{d}\right| \leq \eta_{d}$ and $\left|\psi_{q}\right| \leq \eta_{q}$.

\section{Sliding Mode Controller}

Assuming the following SM switching surfaces:

$$
\begin{aligned}
S(t) & =\left[\begin{array}{l}
s_{d}(t) \\
s_{q}(t)
\end{array}\right]=\left[\begin{array}{l}
\gamma \int_{0}^{t} e_{d}(\tau) d \tau \\
\gamma \int_{0}^{t} e_{q}(\tau) d \tau
\end{array}\right] \\
& =\gamma \int_{0}^{t}\left[\begin{array}{l}
e_{d}(\tau) \\
e_{q}(\tau)
\end{array}\right] d \tau=\gamma \int_{0}^{t} e(\tau) d \tau
\end{aligned}
$$

where:

$S(t)=\left[\begin{array}{l}s_{d}(t) \\ s_{q}(t)\end{array}\right], e(t)=V_{t}-V_{t}^{*}=\left[\begin{array}{l}e_{d}(t) \\ e_{q}(t)\end{array}\right]$

$e_{d}=v_{t d}-v_{t d}^{*}$ and $e_{q}=v_{t q}-v_{t q}^{*}$ represent the tracking errors and $\gamma$ is positive constant. Differentiating (19) with respect to time ( $t$ ) and using equitation (17), yields:

$$
\dot{S}(t)=\gamma e(t)=\gamma\left(V_{t}-V_{t}^{*}\right)=\gamma Y+\gamma \psi-\gamma V_{t}^{*}
$$

Based on Lyapanouv theory, using (21), the SM controllers can be obtained as:

$$
\begin{aligned}
& y_{d}=v_{t d}^{*}-\hat{\eta}_{d} \operatorname{sgn}\left(s_{d}(t)\right) \\
& y_{q}=v_{t q}^{*}-\hat{\eta}_{q} \operatorname{sgn}\left(s_{q}(t)\right) \\
& \left\{\begin{array}{l}
\dot{\hat{\eta}}_{d}(t)=k\left|s_{d}(t)\right| \\
\dot{\hat{\eta}}_{q}(t)=k\left|s_{q}(t)\right|
\end{array}\right.
\end{aligned}
$$


where $\hat{\eta}_{d}$ and $\hat{\eta}_{q}$ are the estimated values of $\eta_{d}$ and $\eta_{q}$; and $k$ is positive constants, and $\operatorname{sgn}(\cdot)$ is sign function. Define a Lyapunov function as:

$$
L(t)=\frac{1}{2}\left(s_{d}^{2}(t)+s_{q}^{2}(t)\right)+\frac{1}{2 k}\left(\tilde{\eta}_{d}^{2}(t)+\tilde{\eta}_{q}^{2}(t)\right)
$$

where $\quad \tilde{\eta}_{d}(t)=\hat{\eta}_{d}(t)-\eta_{d} \quad$ and $\quad \tilde{\eta}_{q}(t)=\hat{\eta}_{q}(t)-\eta_{q}$. Derivativing (25) with respect to the time $(\mathrm{t})$, linking (21), (22) and (23), it is resulted that:

$$
\begin{aligned}
\dot{L}(t) & =S_{d}(t) \dot{S}_{d}(t)+S_{q}(t) \dot{S}_{q}(t)+\frac{1}{k}\left(\tilde{\eta}_{d}(t) \dot{\tilde{\eta}}_{d}(t)+\tilde{\eta}_{q}(t) \dot{\tilde{\eta}}_{q}(t)\right) \\
= & S_{d}(t) \psi_{d}-\eta_{d}\left|S_{d}(t)\right|+S_{q}(t) \psi_{q}-\eta_{q}\left|S_{q}(t)\right| \\
& \leq\left|S_{d}(t)\right|\left|\psi_{d}\right|-\eta_{d}\left|S_{d}(t)\right|+\left|S_{q}(t)\right|\left|\psi_{q}\right|-\eta_{q}\left|S_{q}(t)\right| \\
& =-\left|S_{d}(t)\right|\left(\eta_{d}-\left|\psi_{d}\right|\right)-\left|S_{q}(t)\right|\left(\eta_{q}-\left|\psi_{q}\right|\right) \leq 0
\end{aligned}
$$

Defining the following function:

$$
P(t) \equiv\left|s_{d}(t)\right|\left(\eta_{d}-\left|\psi_{d}\right|\right)+\left|s_{q}(t)\right|\left(\eta_{q}-\left|\psi_{q}\right|\right) \leq \dot{L}
$$

then:

$$
\int_{0}^{t} P(\tau) d \tau \leq L(0)-L(t)
$$

Since $L(0)$ is bounded and also $L(t)$ is nonincreasing and bounded, therefore:

$\lim _{t \rightarrow \infty} \int_{0}^{t} P(\tau) d t<\infty$

In addition, $P(t)$ is bounded. Therefore from Barbalat's lemma, we have:

$$
\lim _{t \rightarrow \infty} P(t)=0
$$

$t \rightarrow \infty$

It means that $S_{d}(t) \rightarrow 0$ and $S_{q}(t) \rightarrow 0$ as $t \rightarrow \infty$. As a result, the proposed SM flux and speed controllers are asymptotically stable, even if system uncertainties exist. Moreover, the flux-tracking errors $e_{d}$ and $e_{q}$ will converge to zero.

The PLL provides the basic synchronizing signal which is the phase angle of the bus voltage, $\theta$. It is obtained from the zero crossing of the bus voltage.

\section{Simulation Results}

The system is simulated in order to validate the controller performances. The effectiveness and validity of the controller system is supported by computer simulation.
The overall block diagram of the proposed control system is shown in Figure 3. A PSIM computer program was developed to model this system on P.C.

In the system shown in Figure 3, the terminal reference voltages $\left(v_{t a}^{*}, v_{t b}^{*}, v_{t c}^{*}\right)$ are applied to the controller and the $\mathrm{d}$-q terminal reference voltages $\left(v_{t d}^{*}, v_{t q}^{*}\right)$ are obtained. Then the appropriate inverter's output voltage $\left(v_{S d}, v_{S q}\right)$ is calculated by Sliding Mode controller and then is produced by PWM inverter to the coupling transformer, in order to obtain the desired terminal voltage $\left(v_{t a}^{*}, v_{t b}^{*}, v_{t c}^{*}\right)$. In this case it is assumed that at $\mathrm{t}=0 \mathrm{sec},\left|v_{t a}^{*}\right|$ is equal to $315 \mathrm{v}$ and rises down to $325 \mathrm{v}$ at $\mathrm{t}=0.2 \mathrm{sec}$. A vector control is applied to the control system; it means that d-axis of the synchronous reference frame is fitted on the vector of $v_{t a}^{*}$, then $v_{t d}^{*}=\left|v_{t a}^{*}\right|$ and $v_{t q}{ }_{t q}=0$. Simulation results shown in Figure 4 are obtained in the condition of $25 \%$ uncertainty in the power system and coupling transformer parameter and 100\% variation in load impedance and $4 \%$ variation in $E$. In addition, the parameters of the proposed control system are given as follows:

$k=3, \gamma=50$

As shown in Figure 4, $v_{t d}$ rises up from $315 \mathrm{v}$ to $325 \mathrm{v}$ at $\mathrm{t}=0.2 \mathrm{sec}$, so $\left|v_{t a}\right|$ can track $\left|v_{t a}^{*}\right|$ while the variations and uncertainties are present in power system.

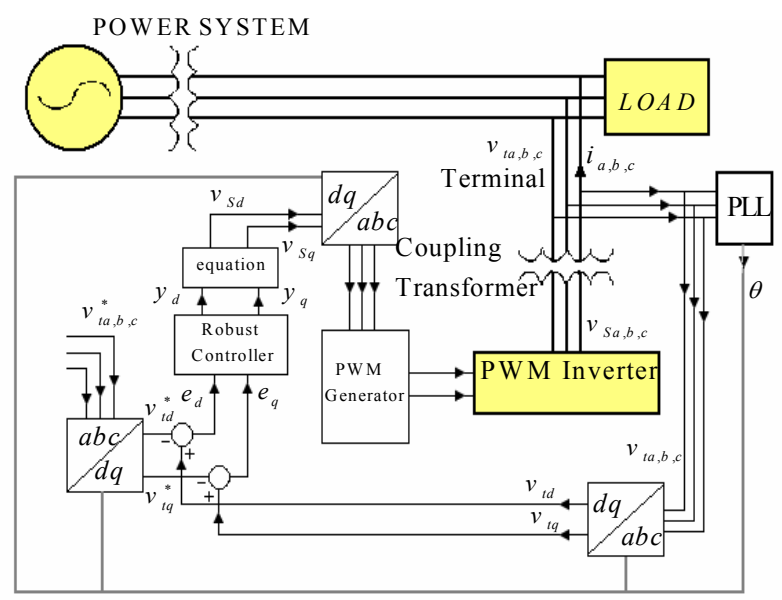

Fig. 3. Simulated block diagram

\section{Conclusions}

A robust controller has been proposed for a PWM Converter connected as a STATCOM. The controller is designed based on Sliding Mode technique. The system control laws are obtained based on Lyaponuv Theory. The system performance Figure 3. Simulated block diagram is verified by computer simulation. Simulation 
results obtained, show that the system is capable of compensation the parameters variations and uncertainties.

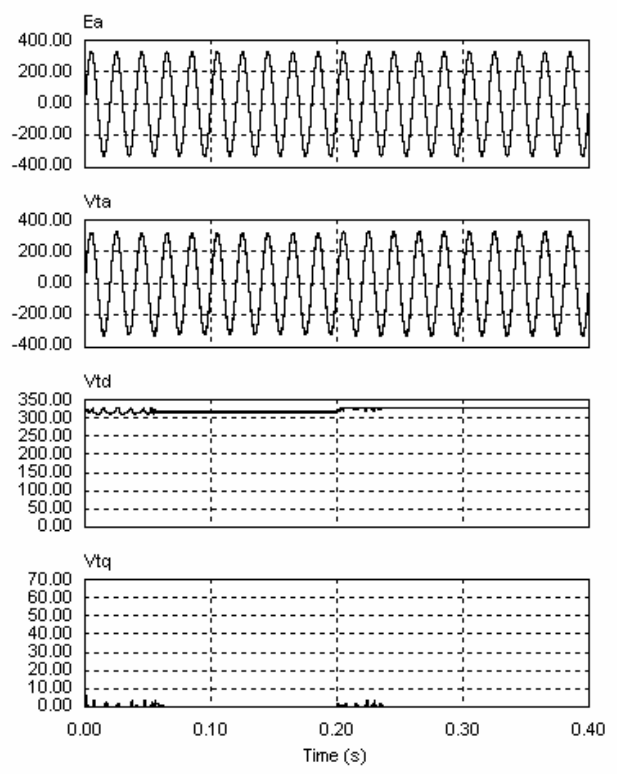

Fig. 4. Simulation Results(Phase a), $E_{a}, v_{a}, v_{t d}, v_{t q}$

\section{References}

[1] L. Guygyi, "Reactive Power Generation and Control by Thyristor Circuits", IEEE Transaction on Industry Applications, Vol.15, No. 5, 1979.

[2] C. W. Edwards, K. E. Mattern, E. J. tacey, P. R. Nannery and J Gubernick, "Advanced Static VAR Generator mploying GTO Thyristors”, IEEEIPES 1988.

[3] L. Gyugyi et al, "Advanced Reactive Power Compensator Using GTO for applications of Electrical Energy distribution", GIGRE 1990, Paris, France.

[4] Z. Yao, N. Lechevin "Issues on Nonlinear Control of Voltage Source Facts Devises", IEEE Proc. On control applications, 2005, Toronto, Canada.

[5] Jain, A.K. Behal, A. Zhang, X.T. Dawson, D.M.Mohan, N.: "Nonlinear controllers for fast Voltage regulation using STATCOMs", IEEE Control Systems Technology, Nov. 2004, pp. 827-842, Vol. 12, No. 6.

[6] Z. Yao, M. S. Dawande and V. Rajagopalan, "InputOutput Linearization for Advanced Reactive Power Compensators", PESC'97, 22-27 June, 1997, pp. 936-41. [7] Z. Yao, V. Donescu, P. Kesimpar, N. Léchevin,V. Rajagopalan, "Nonlinear Control for STATCOM Based on Differential Algebra", 1323 IEEE Power Electronics Specialists Conference-PESC'98, 17-22 May 1998, Fukuoka, Japan.

[8] A. Abbasian, J.Soltani "Robust Nonlinear Control of Linear Induction Motor taking into account the Primary End Effects", IEEE Proc on Industry Applications, Vol. 10, No. 6. 